\title{
Study on the Traffic Organization of Multi-project Construction Period with Three-level Ease Method
}

\author{
Zhanpu Jiao \\ China Railway Siyuan Survey and Design Group Co., LTD., Kunming, Yunnan, 650200
}

Keywords: urban traffic; road construction; three-level ease method; traffic impact zone; traffic organization

\begin{abstract}
With the acceleration of China's urbanization, investment in transportation infrastructure is increasing. In this paper, the traffic influence area of the construction period is divided into three levels, and the design method of traffic organization based on the three-level dissolving method is proposed. In the first-level traffic impact zone, peripheral traffic guidance was designed based on the research of the drivers' detour route selections. In the second-level traffic impact zone, regional traffic diversion was designed through macroscopic road network modeling using OD estimation in Trans CAD software. In the third-level traffic impact zone, node traffic organization was designed according to microscopic node simulating evaluation with VISSM software. Last, this paper chose the Wuhan East Lake High-Tech Development Zone, which is surfing a period with large scale construction as an example. Specific traffic organization plans were made to verify the feasibility of the three-level ease method in the traffic organization during large scale construction period.
\end{abstract}

\section{Introduction}

With the rapid economic development of our country, the level of people's travel has been greatly improved. In order to adapt to the ever-evolving traffic demand, the transportation infrastructure in major cities has also been advanced at an unprecedented rate. Different from the traditional single-item construction traffic organization during the construction period, under the conditions of full study of the traffic impacts of the road construction project and the traffic characteristics of the construction road section, using traffic modeling and simulation and other technical means, a unique construction traffic organization based on the three-level desrosion method was proposed. The design method can provide a more scientific and rational traffic organization plan for large-scale road construction in urban areas.

\section{Determination of the Three-level Traffic Impact Area of the Construction Project}

At present, common model methods include smoke and rain model law, category attraction law, time-based quantization method (T-TIA) and commercial location method, etc. [1]. Based on the determination methods of various traffic-affected areas, this paper proposes the method of determining the three-level traffic-affected areas in the third-level relief method.

In the first-class traffic-affected zone, travel time is a major factor to consider, that is, if the travel time is significantly reduced, a certain degree of detour distance is acceptable. This article uses a time-based quantitative method (T-TIA) to determine the scope of the first-level impact zone. The time-based quantification method is mainly to measure whether the service level of the road network is reduced or reduced by comparing the travel time of the road network before and after the project construction, and can quantify the impact scope more accurately.

In the secondary traffic-affected area, the service level of the road network is the main evaluation index of the affected area. According to the graded description of the service level, the minimum service level that the driver can tolerate is Class D. The road service level at the boundary of the construction affected area is not higher than Grade D as the basis for the need to adopt traffic organization measures. When large-scale construction of multiple projects is carried out 
simultaneously, the superposition of traffic impacts of multiple projects should be considered. This paper adopts TransCAD software to forecast the traffic distribution of regional road network, and obtains macro indicators such as road saturation and service level.

The three-level traffic-affected zone is judged according to the delay, and the construction operation point is mainly related to the way of construction of the fence and the road conditions and line shape of the road, and there is no construction intersection, and the intersection saturation is estimated. In this paper, Vissim simulation software is used to simulate the selected three-level traffic influence area, and the average delay of the output vehicles for different traffic organization plans is determined to determine the optimal traffic organization plan.

\section{Three-level Sparse Traffic Organization Design}

The composition of traffic flow on the construction road is not unique. During the construction period, the traffic organization plan needs to adopt different traffic management measures for different types of traffic flow. The third-level desalination method adopts targeted traffic organization measures for different types of traffic in three different traffic-affected areas, and gradually improves the adverse effects of construction on road traffic. The main contents of the third-level decomposing method can be summarized as follows: first-level peripheral traffic guidance, second-level regional traffic diversion, and third-level node traffic organization.

The path selection model is based on path utility and reflects the driver's travel cost during the driver's choice of path. This paper considers two influencing factors of travel time and detour distance to calculate the cost of different routes under ideal conditions. The driver always tends to choose a route with lower travel cost. The higher the cost of the bypass route compared to the original route, the smaller the driver's willingness to walk.

Where: $\mathrm{C}$ - generalized travel cost;

l — Travel distance;

$\mathrm{t}$ - travel time;

$\alpha$ - weight coefficient $(0<\alpha<1)$;

For different drivers, the weight coefficient $\alpha$ in choosing a trip decision is not the same. When $\alpha$ is set to 1 , the driver's trip cost is completely determined by the travel time. When $\alpha$ is 0 , the driver's trip cost is completely determined. Travel distance; when $\alpha$ is between 0 and 1, the driver's judgment on travel cost has a focus on travel time and travel distance. Therefore, when the road construction, the driver chooses a detour route when facing the traffic guidance will have different results.

OD inversely infers the distribution matrix of OD according to the traffic volume of the road segment, which is the reverse process of traffic allocation. Through research and comparison, we found that the OD matrix backstepping procedure based on stochastic user balancing method is better, because the stochastic user balancing method allows the use of less attractive paths, and the distribution results are closer to the actual traffic observed on the road segment [2].

The traffic flow forecast result is the result of traffic balance after road construction. In real life, after the start of road construction, it is impossible for road traffic to reach equilibrium. Relying on the adjustment of traffic flow to achieve this state of balance requires a process that may cause traffic congestion for a long time, and the purpose of regional traffic organization is to speed up The speed of traffic flow balance. On the basis of traffic flow forecasting, through analysis of road network service levels after construction and before construction, the secondary traffic impact area is defined, that is, the area where traffic organization measures need to be implemented. When the service level of the road is affected by the construction and is lower than the D-class service level, the road section and its adjacent roads and major intersections are secondary traffic impact areas. In the secondary traffic impact area, corresponding traffic organization measures are formulated according to traffic flow direction predicted by the traffic.

The objective of regional traffic diversion is mainly the traffic in the neighborhood of the traffic flow in the construction section. The main measures for regional traffic diversion are: 1 Road network alternative road diversion; 2 road traffic control: banned goods, prohibited, one-way traffic, 
and tidal traffic.

In the construction of multiple construction projects within the area, the projects will affect each other and produce an additive effect. Therefore, it is necessary to reasonably plan the project projects in the region. The construction organization must follow two principles: 1 in the same period (when multiple project constructions are in the same travel path, and the project should be arranged for construction in the same period); 2 parallel stages (when multiple projects are being constructed Take a parallel approach to travel, and multiple construction projects will be staged.)

The traffic simulation of nodes is mainly for small-scale micro-regions. When simulating the road traffic flow in VISSIM, the car-following model adopts a "psychological-physiological follow-up model”, and the lane change model is a rule-based algorithm. Using VISSIM traffic simulation software to delay the simulation of the traffic node evaluation, through the acquisition of node geometry, lane number, canalization, traffic volume and signal timing and other information, output node travel time, delay time, queue length and other indicators.

The measures to optimize the traffic organization in the three-tier traffic-affected areas (nodes) mainly include: 1) traffic signal control (according to the changes in the amount of traffic after the construction of the corresponding signal control); 2) intersection channelization (intersection widening lane, setting up canalization Islands, guardrails, traffic markings, etc.; 3) traffic management facilities in the construction work area (fences, road barriers, cone-shaped traffic signs, construction warning lights, etc.); 4) emergency security measures (Association police on duty, configuration of trailers)

In the three-level traffic-affected zone, micro-level traffic behavior is considered. At this time, traffic not only has cars, but also needs to organize traffic and pedestrian traffic in the construction area.

\section{Take the Construction of Multi-project Construction in Wuhan East Lake High-tech Zone as an example}

In the past two years, a number of municipal construction projects in Donghu Hi-tech Development Zone in Wuhan have started. Among them are the Optics Valley Plaza complex [3], the Optics Valley Avenue rapid transformation [4], the rail transit second line south extension line [5] and other key projects.

Optical Valley Plaza Complex: An integrated rail transit project, municipal engineering, and underground public space. Including rail transit Line 2, Line 9, Line 11 of the interval site, municipal roads Shuyu Road, Lumo Road underpass tunnels and the use of stations, tunnels excavation designed underground public space.

Guanggu Avenue rapid transformation: Donghu High-tech Zone planning one of the major north-south traffic artery, the project from the East Lake Channel exit - Yujiashan North Road, south to the optical valley financial port, the entire two-way six-lane elevated, full-length $7.7 \mathrm{~km}$.

Rail transit South Line No. 2 line: Take the terminal station of the second phase of the first phase of the project from the Guanggu Plaza Station as the starting point, along the route passing Yuxiong Road, Guanshankou, Guanggu Avenue, Jiayuan Road, Nanhu Avenue, Dashudong Road, Gaoxinsi Road, Gaoxinliu Road, Canglong East Street, Buddha Ridge Station, etc., to Buddha Ridge parking lot, a total length of 13.44 kilometers.

From the construction project location, it can be seen that the roads affected by the construction mainly include Luoyu Road, Lumo Road, Guanggu Avenue and Gaoxin Liu Road. In conjunction with the surrounding road network, passing through the traffic zone of Luoyu Road and Guanggu Plaza, the optional travel routes at the street entrance are:

The original route: Yu-Yu West Road (Main Road)-Guanggu Plaza--Yu-Yu East Road (main road);

Detour path 1: Luanshi Road (fast road) - Xiongchu Avenue elevated (fast road);

Detour path 2: Lu Shi Road (Express Road + ground auxiliary road) - South Lake Avenue (main road);

Detour path 3: Lu Shi Road (Express Road) - Third Ring Road (Express Road). 
At the intersection of Luoyu Road, the total travel time of each route is calculated by calculating the sum of the travel time and intersection delay of the original route and the other three bypass routes, and the sum of travel time and intersection delay. Calculate the travel distance between the original path and the other three detour paths from the map. The results are shown in the following table:

Table 1 Travel time of each path in Jiedaokou

\begin{tabular}{ccccc}
\hline path & $\begin{array}{c}\text { Road section } \\
\text { driving time }\end{array}$ & $\begin{array}{c}\text { Intersection } \\
\text { delay }\end{array}$ & Travel time & $\begin{array}{c}\text { Travel } \\
\text { distance }\end{array}$ \\
\hline Original path & $23 \mathrm{~min}$ & $8 \mathrm{~min}$ & $31 \mathrm{~min}$ & $9.3 \mathrm{~km}$ \\
$\begin{array}{c}\text { Detour path } \\
\begin{array}{c}\text { Detour path } \\
\text { two }\end{array}\end{array}$ & $15 \mathrm{~min}$ & $6 \mathrm{~min}$ & $21 \mathrm{~min}$ & $10.7 \mathrm{~km}$ \\
$\begin{array}{c}\text { Detour path } \\
\text { three }\end{array}$ & $22 \mathrm{~min}$ & $10 \mathrm{~min}$ & $33 \mathrm{~min}$ & $13.5 \mathrm{~km}$ \\
\hline
\end{tabular}

The detour path 1 and the detour path 3 have obvious advantages in terms of travel time, but the travel path 3 has a slightly longer travel distance, and the detour path 2 has little difference with the original path in the travel time and the detour distance. Therefore, traffic-induced signs need to be set up on the north and east of the street intersection to indicate that the vehicle will detour to the Second Ring Road and the Third Ring Road. Based on the feasibility of the detour route, it is determined that the first-class traffic-affected zone is a closed area such as the Second Ring Road, the Third Ring Road, the Guanggu Second Road, and the High-New Road and six roads. In the first-level impact area, 16 traffic guidance signs are set up at the inducing node.

According to the characteristics of the project construction, traffic flow observations were conducted on the construction sections, adjacent main roads, secondary arterial roads and important intersections of the three project projects. After investigating the traffic volume of major roads, in order to simulate the traffic volume of the entire road network, it is necessary to use the OD inverse function in TransCAD. This function can be used to infer the current OD matrix, and can also be allocated similar to the current traffic volume. Traffic allocation results.

After the road network was established, OD reversed the process at TransCAD. To better approximate the true OD, an OD matrix estimated from traffic conditions is used as the original OD matrix.

According to the construction process of the construction project, calculate the capacity of the construction period, and modify the relevant attributes of the road network during the construction period of the project in TransCAD. Based on the inversely launched OD matrix as the base OD matrix, the traffic distribution operation of the road network is performed using the user balance method, and the traffic flow of the road network during the construction period is predicted.

Compared with the traffic volume of the road network before construction, the traffic volume of construction sections such as Guanggu Avenue, Luoyu Road and Gaoxinliu Road decreased, and V/C increased slightly. Traffic on Guanshan Avenue, Guanggu Yilu Road and Xiongchu Avenue (Gaoxin Avenue) increased. Therefore, the D-level service level road outside the construction section is the boundary of the secondary impact area, and the secondary traffic diversion signs are placed at the intersections of the diverted roads such as Guanshan Avenue, Guanggu Yilu Road and Xiongchu Avenue (Gaoxin Avenue). Divert traffic ahead of time to avoid entering congested roads. In addition, large-scale vehicles are diverted, and large-scale trucks are prohibited from entering the roads at Yuyu East Road and Guanggu Avenue, and large trucks bypass the Guanggu Road.

Construction Organization Strategy:

Jiayuan Road Station is located at the intersection of Optics Valley Venture Street and Jiayuan Road. The construction needs to block the north-south traffic of Jiayuan Road. The rapid transformation of adjacent Optics Valley Avenue will reduce the capacity of Guanggu Avenue. The two construction projects are in parallel. State, will bring enormous pressure to the local north-south 
direction of traffic, in the case of construction time, the construction time of the two projects should be staggered as far as possible, in the Optics Valley Avenue to restore certain traffic capacity after the closure of Jiayuan Road.

Guanggu Avenue, the intersection of Lingjiashan North Road, was used to invade the interior of the intersection. After the block, the south and north entrances of Guanggu Avenue were two lanes, and an intersection simulation model was established.

From the simulation results, it can be seen that the two north and south nodes of the node have a long delay in the direction of entry. The number of queuing vehicles is close to 100, and vehicles cannot pass through. According to the analysis of the results, the node's two lanes of north and south entrances cannot meet the traffic demand and need to be widened. The south entrance construction retaining wall is contracted at the intersection, and it can be widened into three lanes. The construction of the north entrance is restricted. Consider the opening of the fence and use the enclosure as a channelization island to form a right turn lane.

In accordance with the optimized plan, the intersection simulation model was reconstructed, signal control was adjusted, node traffic organization was simulated, and node evaluation results were output. In addition to the large amount of left turn left in the southbound lane of the South Entrance, there is still a long queue, and the delays and queuing lengths of the Guanggu Avenue in both the north and the south are significantly reduced.

Due to the limitation of construction conditions, under the condition that there is no feasible channel to increase the left-turn lane of the south entrance of the node, the left-turn traffic pressure of this node is still relatively large. Therefore, during the peak period, the police officer needs to be set on duty to divert traffic. Set up various types of construction management facilities as required, and formulate a node traffic organization plan.

\section{Conclusion}

This article describes the scope of traffic impacts of construction projects, and classifies the three-tier traffic-affected areas according to the characteristics of the three-level dredging method. At the same time, it also proposes different traffic impact assessment indicators for the three-tier traffic impact areas. Based on the study of the driver's bypass selection, the use of TransCAD to perform OD back-propagation for macro-traffic modeling and the use of VISSIM for microscopic traffic simulation of nodes, the specific organizational measures for road traffic during construction are proposed in different levels. The traffic traffic organization design method based on the three-tier traffic debriefing method based on the first-level peripheral traffic guidance, the second-level regional traffic diversion, and the three-level node traffic organization was obtained. In combination with the practical application of the three-level desorption method, traffic organization design examples are analyzed for large-scale construction of many engineering projects in reality, and traffic organization design plans that meet actual conditions are proposed. Practice shows that the three-level dredging method has a clear hierarchy and traffic organization measures are highly targeted, and it has good practicality for large-scale construction.

\section{References}

[1] Qian Jun, Yuan Chunling, Yang Lulu. Comparison of methods for determining the scope of traffic impact[J]. Science of Communications Technology and Economy. 2012, 1: 45 48.

[2] Wei Xianlan, Li Haifeng, Yan Yanbin et al. Comparative Study of OD Matrix Backdating Results on TransCAD Platform [J]. Highway. 2007, 6: 122 128.

[3] Huazhong University of Science and Technology Transportation Laboratory. Wuhan Optics Valley Plaza Complex Transportation Organization [R]. Wuhan: Huazhong University of Science and Technology Traffic Laboratory, 2015.

[4] Traffic Laboratory of Huazhong University of Science and Technology. Transportation organization during construction of Guanggu Avenue rapid reconstruction project [R]. Wuhan: 
Traffic Laboratory of Huazhong University of Science and Technology, 2015.

[5] Traffic Laboratory, Huazhong University of Science and Technology. Transportation Organization during the Construction Period of the South Extension Line of Wuhan Metro Line 2 [R]. Wuhan: Traffic Laboratory, Huazhong University of Science and Technology, 2016. 